Supplementary Information

\title{
In Silico Insight into the Reductive Nitrosylation of Ferric Hemeproteins
}

\author{
Nicolás O. Foglia, ${ }^{\dagger, \dagger}$ Sara E. Bari,,$^{\dagger}$ Dario A. Estrin $*, \dagger, \dagger$ \\ † Universidad de Buenos Aires. Consejo Nacional de Investigaciones Científicas y \\ Técnicas. Instituto de Química de los Materiales, Medio Ambiente y Energía. Facultad de \\ Ciencias Exactas y Naturales, Buenos Aires, Argentina. \\ † Universidad de Buenos Aires. Facultad de Ciencias Exactas y Naturales. Departamento \\ de Química Inorgánica, Analítica y Química Física. Buenos Aires, Argentina
}

Corresponding Author: dario@qi.fcen.uba.ar 
SI-1. Dimensions of the water box: the dimensions of the octahedral water box were 109.56 $\AA 109.56 \AA 109.56 \AA 109.47^{\circ} 109.47^{\circ} 109.47^{\circ}$ for swMbFe ${ }^{\mathrm{III}}, 124.54 \AA 124.54 \AA 124.54$ $\AA 109.47^{\circ} 109.47^{\circ} 109.47^{\circ}$ for $\alpha \mathrm{hHbFe}^{\mathrm{III}}$, and $105.74 \AA 105.74 \AA 105.74 \AA 109.47^{\circ}$ $109.47^{\circ} 109.47^{\circ}$ for $\mathrm{RpNP}_{4} \mathrm{Fe}^{\mathrm{III}}$. In the MD simulations the total charge of the system was neutralized using Amber's uniformly charged plasma.

SI-2. Root mean square deviation (RMSD) of the backbone of the HID form of myoglobin versus time, referred to the structure at the first MD frame. The black, red, and green traces correspond to three independent runs.

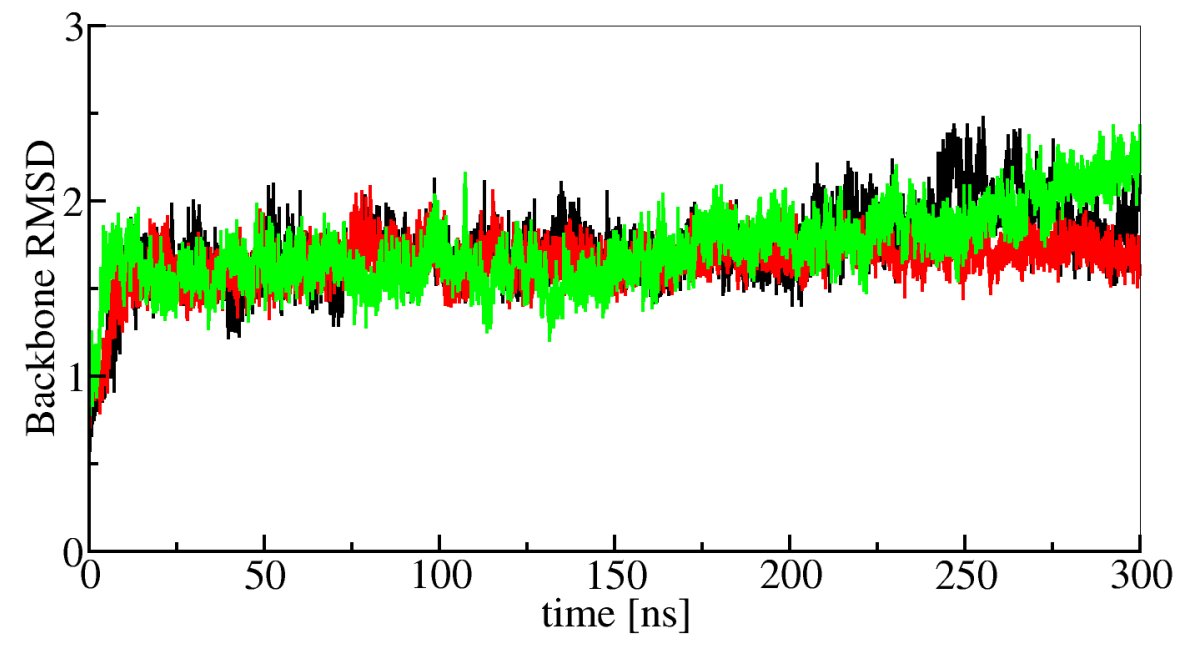

SI-3. Root mean square deviation (RMSD) of the backbone of the HIE form of myoglobin versus time, referred to the structure at the first MD frame. The black, red, and green traces correspond to three independent runs.

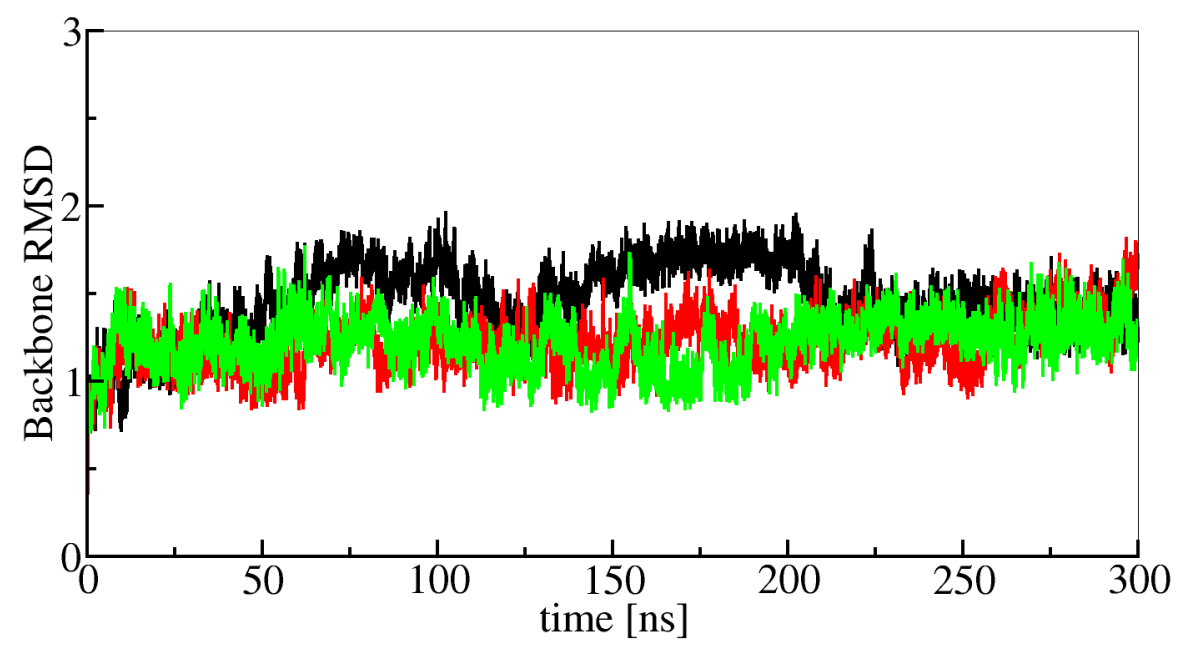


SI-4. Root mean square deviation (RMSD) of the backbone of the HIP form of myoglobin versus time, referred to the structure at the first MD frame. The black, red, and green lines correspond to three independent runs.

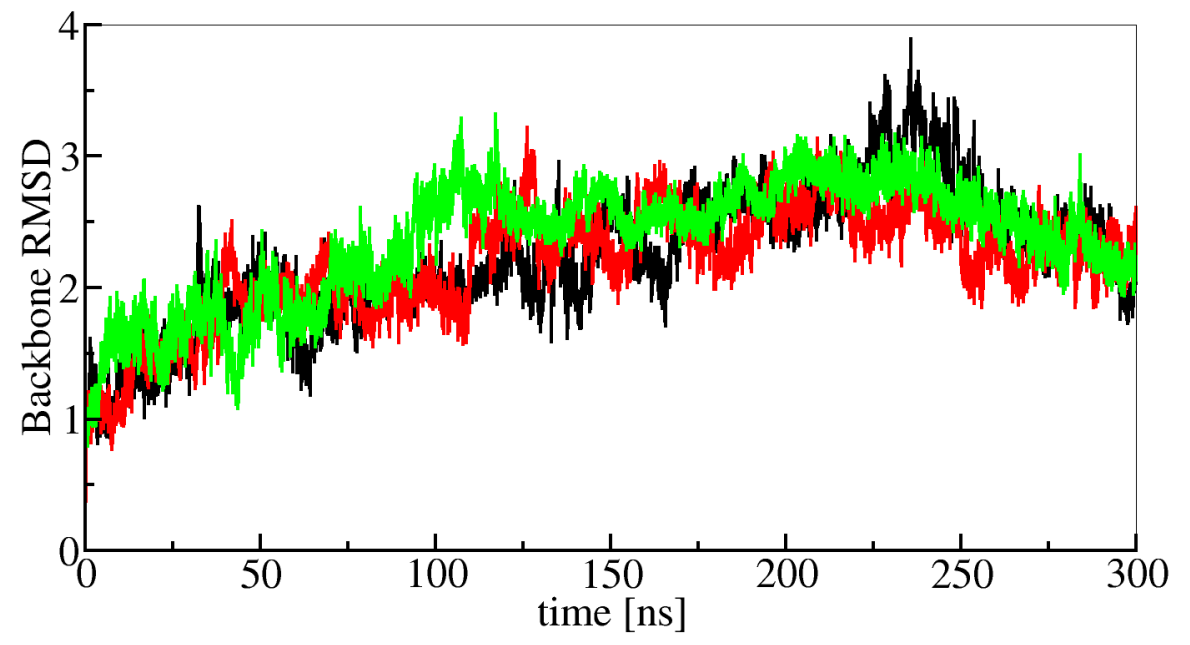

SI-5. Root mean square deviation (RMSD) of the backbone of hemoglobin versus time, referred to the structure at the first MD frame. The black, red, and green lines correspond to three independent runs.

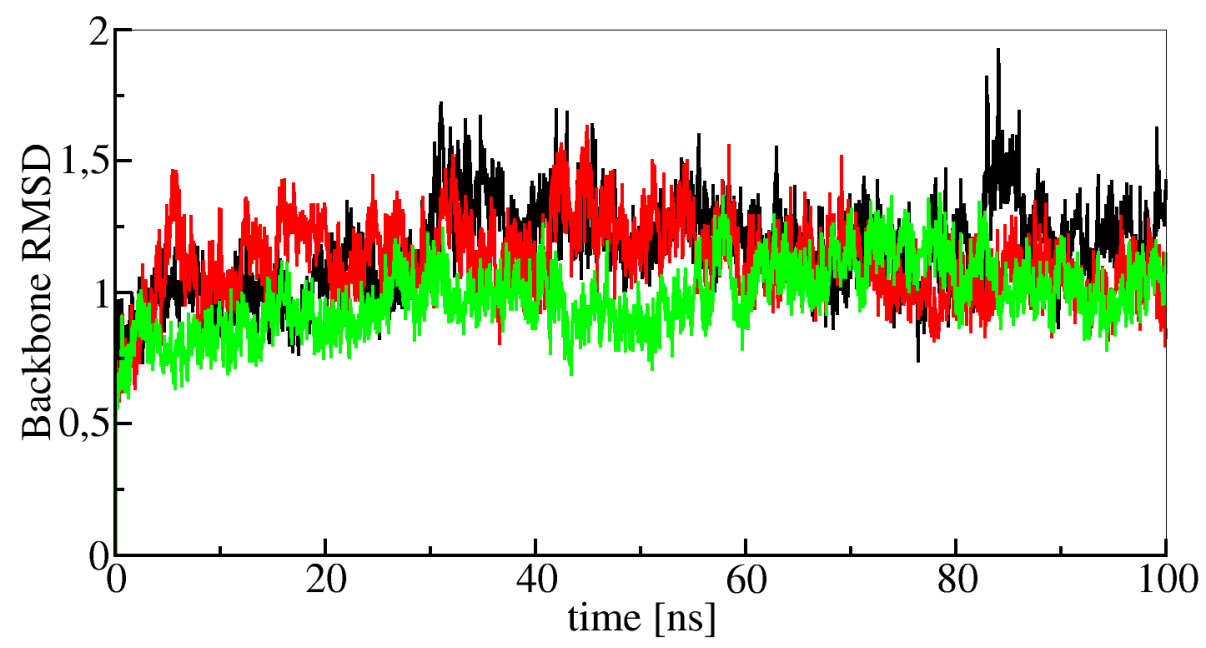

SI-6. Root mean square deviation (RMSD) of the backbone of nitrophorin versus time, referred to the structure at the first MD frame. The black, red, and green lines correspond to three independent runs. 


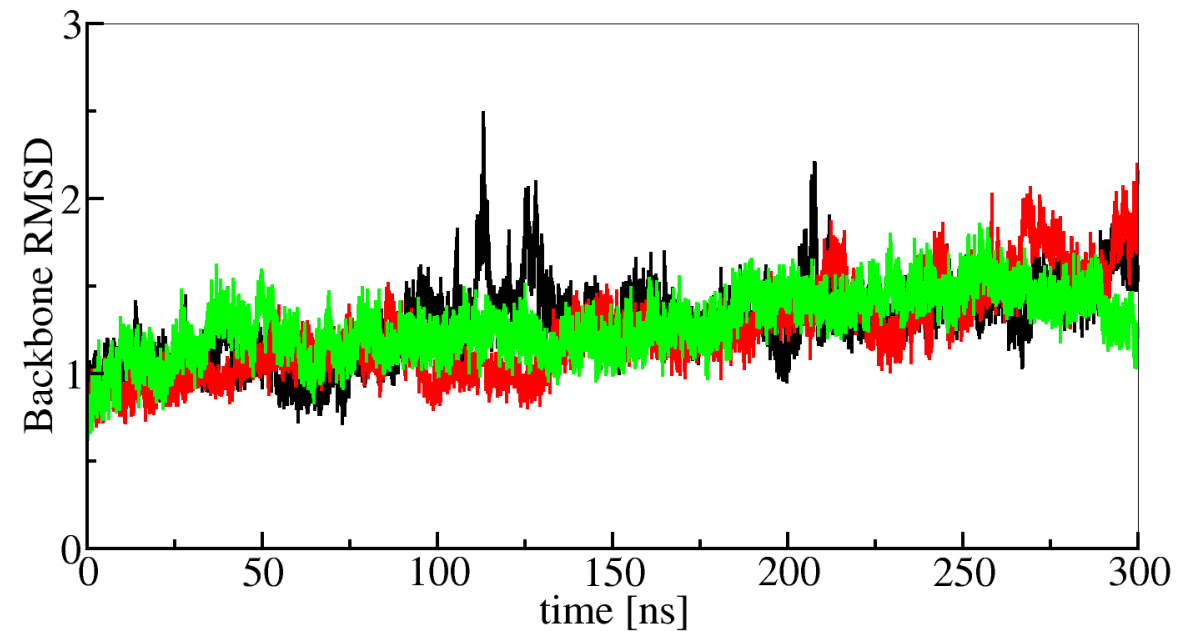

SI-7. Comparison of PBE/DZVP data for the $\mathrm{Fe}^{\mathrm{III}}$-NO and $\mathrm{Fe}^{\mathrm{II}}-\mathrm{NO}$ states of swMb, with geometrical parameters derived from crystallographic or computational data.

\begin{tabular}{|c|c|c|c|}
\hline & \multicolumn{3}{|c|}{$\mathrm{d}$ Fe-N(NO), $[\AA]$} \\
\hline & PBE/DZVP & $\mathrm{PDB}^{2}$ & UB3LYP/VDZ/3-21G \\
\hline $\mathrm{swMbFe}^{\mathrm{III}} \mathrm{NO}$ & 1.65 & na & 1.65 \\
\hline $\mathrm{swMbFe}^{\mathrm{II}} \mathrm{NO}$ & 1.74 & 1.79 & 1.81 \\
\hline
\end{tabular}

\begin{tabular}{|l|c|c|c|}
\cline { 2 - 4 } \multicolumn{1}{c|}{} & \multicolumn{3}{c|}{$\angle \mathrm{FeNO}^{\circ}\left[{ }^{\circ}\right]$} \\
\cline { 2 - 4 } \multicolumn{1}{c|}{} & $\mathrm{PBE} / \mathrm{DZVP}^{1}$ & $\mathrm{PDB}^{2}$ & $\mathrm{UB}^{\mathrm{N}} \mathrm{LYP} / \mathrm{VDZ} / 3-21 \mathrm{G}^{3,4}$ \\
\hline swMbFe $^{\mathrm{III}} \mathrm{NO}$ & 175.1 & $\mathrm{na}$ & 179.9 \\
\hline swMbFe $^{\mathrm{II}} \mathrm{NO}$ & 142.3 & 137.14 & 139.2 \\
\hline
\end{tabular}

${ }^{1}$ Parameters obtained by geometry optimization with $0.04 \mathrm{eV} \mathrm{A}^{-1}$ convergence criteria in forces.

${ }^{2}$ Wang B, Shi Y, Tejero J, Powell SM, Thomas LM, Gladwin MT, Shiva S, Zhang Y, RichterAddo GB. Nitrosyl Myoglobins and Their Nitrite Precursors: Crystal Structural and Quantum Mechanics and Molecular Mechanics Theoretical Investigations of Preferred Fe -NO Ligand Orientations in Myoglobin Distal Pockets. Biochemistry. 2018 Aug 14;57(32):4788-4802.

${ }^{3}$ Nutt DR, Karplus M, Meuwly M. Potential energy surface and molecular dynamics of MbNO: existence of an unsuspected FeON minimum. J Phys Chem B. 2005 Nov 10;109(44):21118-25.

${ }^{4}$ Nutt DR, Meuwly M. Ferric and ferrous iron in nitroso-myoglobin: computer simulations of stable and metastable States and their infrared spectra. Chemphyschem. 2007 Mar 12;8(4):527-36. 
SI-8. Schematic representation of the access of water molecules to the distal site of the $\mathrm{swMbFe}{ }^{\mathrm{III}} \mathrm{NO}$ complex for the three different protonation states of histidine E7. Representative snapshots from the 300ns MD experiments, every $0.05 \mathrm{~ns}$, for the HID, HIE and HIP protonation states.
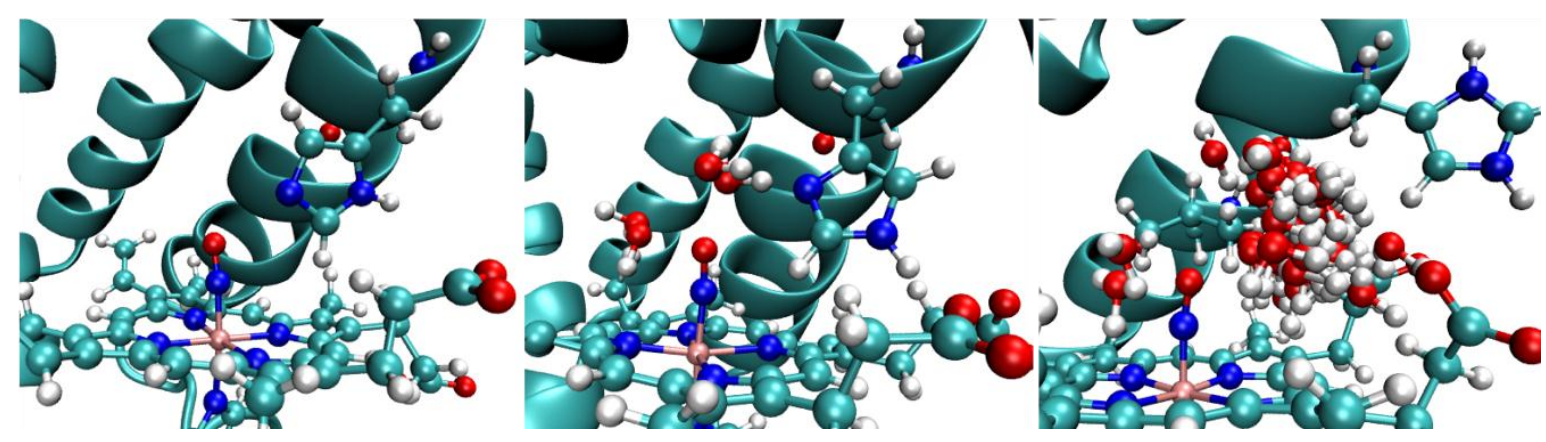

SI-9. Work associated with $\xi_{1}$ and energy profiles for the nucleophilic attack of the $\mathrm{O}\left(\mathrm{H}_{2} \mathrm{O}\right)$ to the $\mathrm{N}(\mathrm{NO})$ of $\mathrm{swMbFe} \mathrm{III}^{\mathrm{III}} \mathrm{NO}$, vs. $\xi_{1}$ as reaction coordinate.

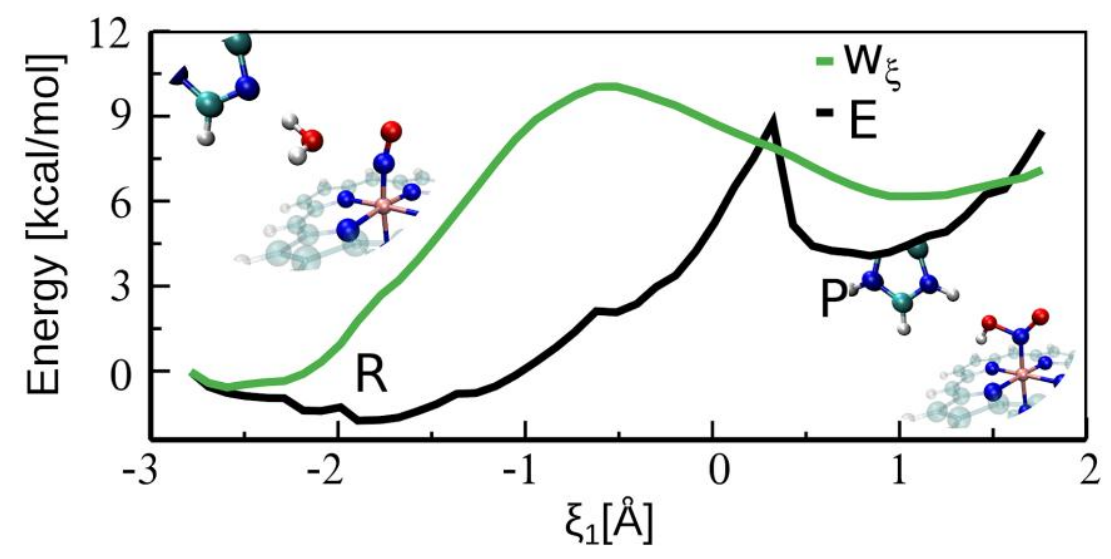

SI-10. Work associated with $\xi_{2}$ and energy profiles for the nucleophilic attack of a $\mathrm{O}\left(\mathrm{OH}^{-}\right)$ to the $\mathrm{N}(\mathrm{NO})$ of $\mathrm{swMbFe}{ }^{\mathrm{III}} \mathrm{NO}$, vs. $\xi_{2}$ as reaction coordinate. 


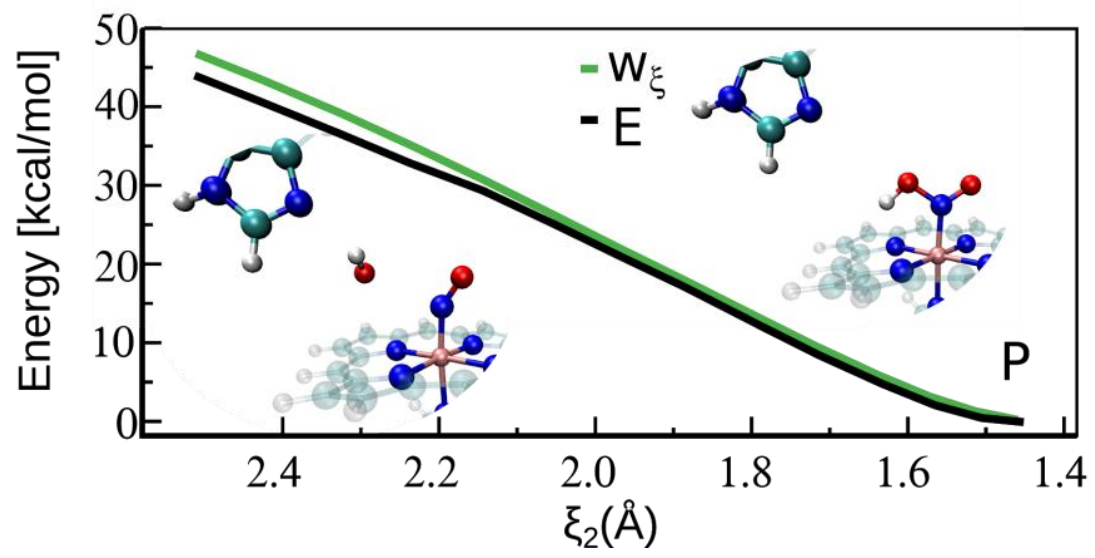

